\title{
Fully Parameterized Macromodeling of S-Parameter Data by Interpolation of Numerator \& Denominator
}

\author{
Dirk Deschrijver, Member, IEEE, and Tom Dhaene, Senior Member, IEEE
}

\begin{abstract}
A robust approach for parametric macromodeling of tabulated frequency responses is presented. An existing technique is modified in such a way that interpolation is performed at the numerator and denominator level, rather than the transfer function level. This enhancement ensures that the poles of the parametric macromodel are fully parameterized. It strengthens the modeling capabilities and improves the model compactness.
\end{abstract}

Index Terms-Parametric Macromodels, Interpolation, Vector Fitting, Rational Transfer Functions, Parameterized Systems.

\section{INTRODUCTION}

$\mathbf{P}$ ARAMETRIC macromodeling techniques are very important for the design, study and optimization of microwave structures and systems. Such macromodels approximate the frequency response of a system that is parameterized by one or more design variables. They are particularly useful for real-time design-space exploration, design optimization and sensitivity analysis. The calculation of such macromodels is not a trivial task, and many different approaches have been investigated, e.g., see [1]-[6] and the references therein.

This letter will focus on one of the techniques that was published recently. In [2], it was proposed to compute a set of one-dimensional macromodels (also called univariate nodes) for different values of the design variables. These nodes are then subsequently combined into a multi-dimensional parametric macromodel by interpolating their input-output port responses with the barycentric interpolation formula [7]. This concept, also known as "transfer function interpolation", has been applied succesfully and several modifications of the modeling approach have been reported in literature [4].

In some specific cases, it was found that transfer function interpolation has some shortcomings which are related to the accuracy and the efficiency of the model. If the poles of the macromodel must travel long distances in the complex plane inbetween the nodes, the response of the model may deviate from the behavior that is expected [5]. Also the efficiency and compactness of the models is not optimal, since the number of poles grows artificially with the number of nodes [6].

The goal of this letter is to address these issues, and to present a solution that is based on the approach in [2]. It is shown that interpolation at the numerator and denominator level leads to a fully-parameterized modeling procedure that is more effective than transfer function interpolation. The

D. Deschrijver and T. Dhaene are with the Department of Information Technology (INTEC), Ghent University - Interdisciplinary Institute for Broadband Technology (IBBT), B-9000 Gent, Belgium (email: dirk.deschrijver@intec.ugent.be; tom.dhaene@intec.ugent.be). This workwas supported by the Research Foundation Flanders (FWO-V). D. Deschrijver is a post-doctoral research fellow of FWO-V. benefits of the approach are demonstrated by applying it to two examples, including a double folded stub filter.

\section{Calculation of the Univariate Nodes}

The parameterized frequency response of a device is characterized by a set of $K$ data samples $\left\{(s, \alpha)_{k}, \mathbf{H}(s, \alpha)_{k}\right\}_{k=1}^{K}$ where $s=j \omega$ represents the frequency variable, $\alpha$ is a design variable, and $\mathbf{H}(s, \alpha)$ is the corresponding transfer function. For $v=1, \ldots, V$ discrete values of the design variable $\alpha$, a set of one-dimensional frequency-dependent macromodels $\mathbf{R}\left(s, \alpha_{v}\right)$ are computed by applying Vector Fitting [8] to the simulated frequency responses. These models, also called univariate nodes in [2], are fitted using the same number of poles. The stability of these models is ensured by means of a simple pole-flipping scheme, and passivity can be enforced a posteriori using various passivity enforcement techniques [9].

\section{TRANSFER FUNCTION INTERPOLATION}

It was proposed in previous work [2] to combine the nodes into a parametric (bivariate) macromodel by interpolating them at the transfer function level with Barycentric interpolation [7]

$$
\mathbf{R}(s, \alpha)=\frac{\sum_{v=1}^{V} \frac{w_{v}}{\alpha-\alpha_{v}} \mathbf{R}\left(s, \alpha_{v}\right)}{\sum_{v=1}^{V} \frac{w_{v}}{\alpha-\alpha_{v}}}
$$

An interesting property of interpolation formula (1) is that it provides a rational interpolation of the nodes as a function of $\alpha$, for any choice of non-zero weights $\left(w_{v} \neq 0\right)$ [7]. A closer inspection of (1), however, reveals that the parametric macromodel is in fact an $\alpha$-weighted sum of the nodes. Hence, the evaluation of (1) for intermediate values of $\alpha$ yields a higher-order macromodel that contains all the poles of all the nodes. Since these poles are fixed (i.e., not parameterdependent), the model response inbetween the nodes can sometimes appear different from the response that is normally expected [5]. In some cases, these two drawbacks may limit the accuracy and efficiency of the modeling approach [6].

\section{NUMERATOR/DENOMINATOR INTERPOLATION}

This section presents a modified approach that solves the difficulties mentioned in Section III, by applying Barycentric interpolation to the numerator and denominator polynomials. For notational convenience, each element on row $m$ and column $n$ of transfer matrix $\mathbf{R}\left(s, \alpha_{v}\right)$ is denoted as $R_{m n}\left(s, \alpha_{v}\right)$. 


\section{A. Macromodel Representation}

Since each matrix element $R_{m n}\left(s, \alpha_{v}\right)$ is modeled by a partial fraction expansion, it can easily be written as the ratio of a numerator $N_{m n}\left(s, \alpha_{v}\right)$ and monic denominator $D_{m n}\left(s, \alpha_{v}\right)$. Rather than applying Barycentric interpolation to the input-output transfer function, it is proposed to apply the same procedure to numerators and denominators individually,

$$
R_{m n}(s, \alpha)=\frac{N_{m n}(s, \alpha)}{D_{m n}(s, \alpha)}=\frac{\frac{\sum_{v=1}^{V} \frac{w_{v}^{N}}{\alpha-\alpha_{v}} N_{m n}\left(s, \alpha_{v}\right)}{\sum_{v=1}^{V} \frac{w_{v}^{N}}{\alpha-\alpha_{v}}}}{\frac{w_{v}^{D}}{\alpha-\alpha_{v}} D_{m n}\left(s, \alpha_{v}\right)}
$$

It is found that this approach does not artificially increase the number of poles, since $D_{m n}(s, \alpha)$ in (2) constitutes an $\alpha$ weighted sum of denominator polynomials that have the same degree. Another clear advantage over the approach in (1) is that the denominator polynomial $D_{m n}(s, \alpha)$, and consequently also the poles of the model, become parameter-dependent in terms of $\alpha$. This means that the poles of model (2) are fully parameterized, as opposed to the fixed-pole solution of (1).

\section{B. Simplified Model Expression}

For most physical systems, the response of the models $R_{m n}(s, \alpha)$ varies smoothly when the value of a design variable $\alpha$ is changed. Therefore, the barycentric weights in (2) are usually chosen in such a way that the interpolation in terms of $\alpha$ is polynomial rather than rational. In this case, the weights $w_{v}^{N}$ and $w_{v}^{D}$ in (2) are computed solely from the parameter values $\alpha_{v}$ of the nodes [7]. Therefore, one can choose $w_{v}$ as

$$
w_{v}=w_{v}^{N}=w_{v}^{D}=\prod_{k=1, k \neq v}^{V}\left(\alpha_{v}-\alpha_{k}\right)^{-1}
$$

The use of common weights $\left(w_{v}^{N}=w_{v}^{D}\right)$ allows the simplification of interpolation formula (2) into an elegant expression

$$
R_{m n}(s, \alpha)=\frac{\bar{N}_{m n}(s, \alpha)}{\bar{D}_{m n}(s, \alpha)}=\frac{\sum_{v=1}^{V} \frac{w_{v}}{\alpha-\alpha_{v}} N_{m n}\left(s, \alpha_{v}\right)}{\sum_{v=1}^{V} \frac{w_{v}}{\alpha-\alpha_{v}} D_{m n}\left(s, \alpha_{v}\right)}
$$

Note that this compact representation is closely related to [1]

\section{EXAMPLE: 2-POLE MACROMODEL FROM [5]}

As a first example, a parameterized 2-pole macromodel is taken from [5], which illustrates the shortcomings of the transfer function interpolation scheme. The macromodel is defined as $H(s, \alpha)=\left(s^{2}+0.01 s+1 \pm \alpha\right)^{-1}$, and its response is considered as a function of parameter $\alpha \in[-0.1,0.1]$ over the frequency range $\omega \in[0.5,1.5]$. Both schemes in Section

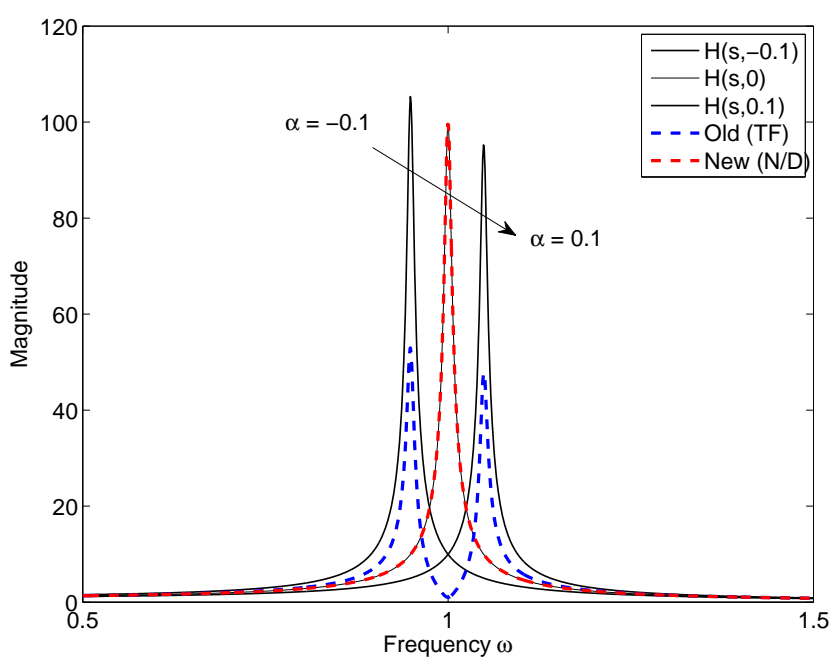

Fig. 1. Parameterized 2-pole macromodel

III and IV are used to interpolate two nodes, $R(s,-0.1)$ and $R(s, 0.1)$, and the model response is compared at intermediate values of $\alpha$, e.g., at the midpoint $\alpha=0$. As shown in Fig. 1, an interpolation of the transfer matrix (blue curve) yields a 4-pole macromodel with $R(s, 0)=(R(s,-0.1)+R(s, 0.1)) / 2$ that deviates strongly from the true model response. By interpolating the numerator and denominator polynomials separately (red curve), the model is able to fit the movement of the peak with an excellent accuracy using only 2 poles. Note that this also corresponds to the correct model order of $H(s, 0)$.

\section{Example: Double Folded Stub Filter}

As a second example, the reflection coefficient, $S_{11}$, and transmission coefficient, $S_{21}$, of a double folded stub microwave filter are modeled using the new interpolation scheme. A parameterized macromodel is computed as a function of the frequency, $f \in[5,20] \mathrm{GHz}$, and a varying stub length, $\alpha \in[2,3] \mathrm{mm}$, as shown by the layout in Fig. 2. The substrate has a relative permittivity of $\varepsilon_{r}=9.9$ and a thickness of 0.127 $\mathrm{mm}$, while the spacing $S$ between the stubs is set to 0.175 $\mathrm{mm}$. The frequency response is simulated for 8 different values of the stub length $\alpha$, which are equidistantly spread over the parameter range of interest. These frequency responses are subjected to the Vector Fitting procedure in order to compute several 9-pole univariate nodes. Fig. 3 and 4 show the response

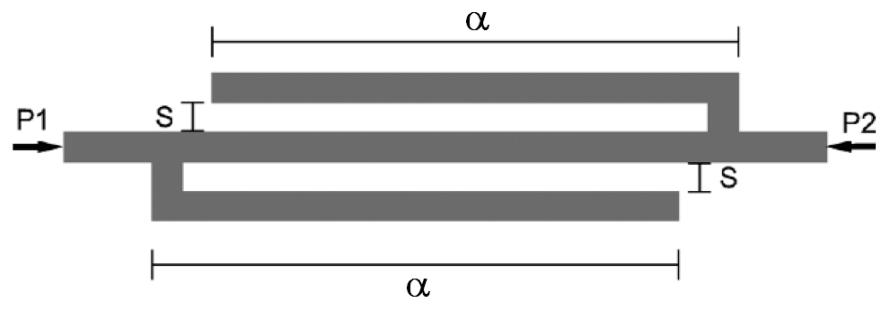

Fig. 2. Layout Double Folded Stub Filter 


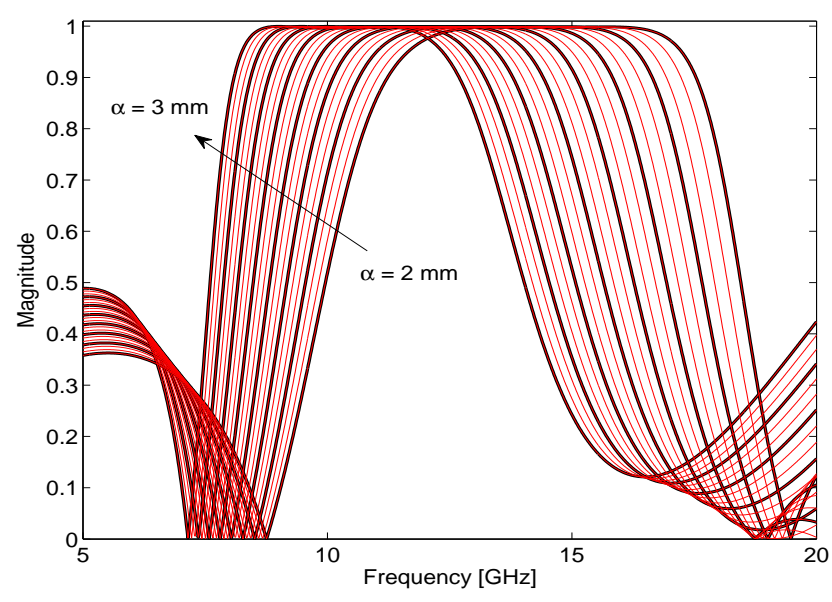

Fig. 3. Magnitude of $S_{11}$ as function of frequency and length $\alpha$ (8 nodes)

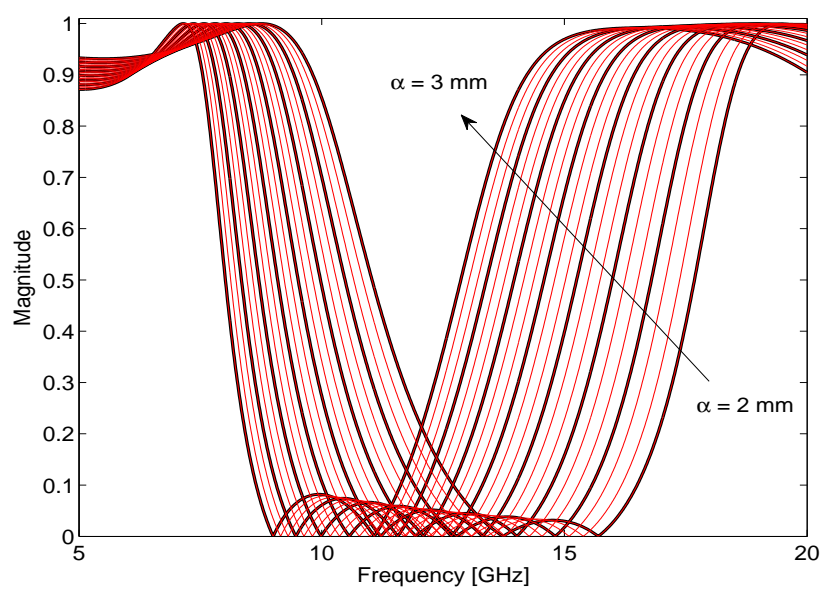

Fig. 4. Magnitude of $S_{21}$ as function of frequency and length $\alpha$ (8 nodes)

of the parameterized macromodel when evaluated over a dense set of parameter values. The bold (black) lines match with the frequency response of the nodes, whereas the thin (red) lines inbetween them are interpolated responses of the model. It is shown that the model is able to capture the dynamical behavior of the data and the movement of the resonances in an accurate way. Fig. 5 visualizes the trajectories of the poles, which are found by computing the roots of $D_{m n}(s, \alpha)$ in (2) or $\bar{D}_{m n}(s, \alpha)$ in (4) for several discrete values of $\alpha$. For any value of $\alpha$, the number of macromodel poles is at most 9 , and does not grow with the number of nodes $V$, as desired.

\section{DISCUSSION}

A multivariate extension of the approach is straightforward, since the barycentric interpolation formula can be applied recursively to the numerators and denominators of lowerdimensional models (i.e., $N$ and $D$ in (2) or $\bar{N}$ and $\bar{D}$ in (4)), as in Section III-C of [2]. Future work will focus on stability and passivity enforcement of the parameterized macromodels (2) and (4) by using specific interpolation schemes [10] and/or

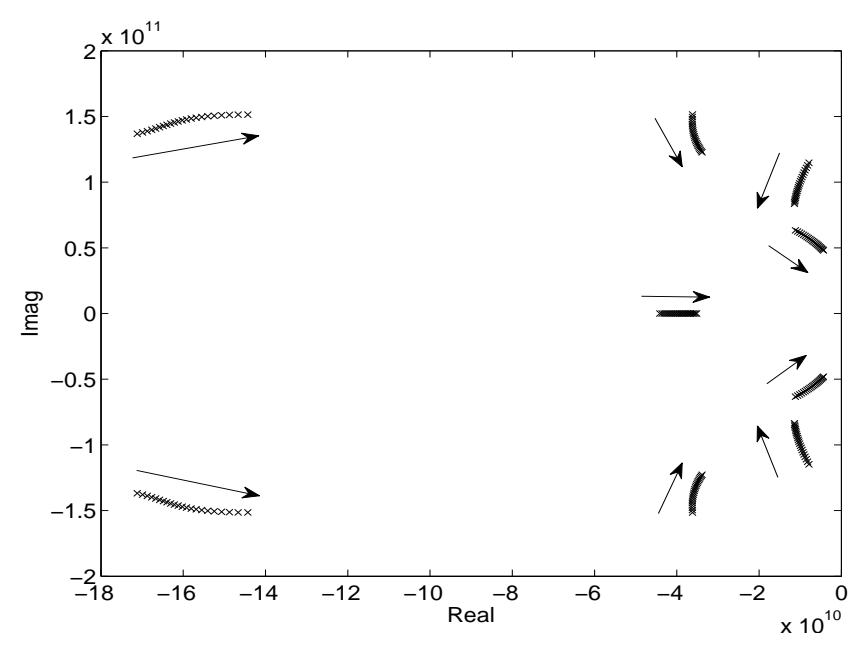

Fig. 5. Trajectories of the parameterized poles $(\times)$ as function of length $\alpha$

applying perturbations to the barycentric weights (i.e., $w_{v}^{N}$ and $w_{v}^{D}$ respectively in (2), or $w_{v}$ in (4)) as proposed in [2].

\section{CONCLUSION}

A modified interpolation scheme is proposed for parametric macromodeling of frequency-domain responses. The method starts by computing several univariate macromodels for different instances of a design variable. These models are then combined into a multivariate macromodel by interpolating the numerator and denominator polynomials, rather than the transfer function. It is shown that this approach avoids pole redundancies in the model, and improves the interpolation capabilities of the macromodel inbetween the nodes [2].

\section{REFERENCES}

[1] D. Deschrijver, T. Dhaene and D. De Zutter, "Robust Parametric Macromodeling using Multivariate Orthonormal Vector Fitting", IEEE Trans. Microw. Theory Tech., vol. 56, no. 7, pp. 1661-1667, July 2008.

[2] D. Deschrijver and T. Dhaene, "Stability and Passivity Enforcement of Parametric Macromodels in Time and Frequency Domain", IEEE Trans. Microw. Theory Tech., vol. 56, no. 11, pp. 2435-2441, November 2008.

[3] P. Triverio, S. Grivet-Talocia and M. S. Nakhla, "A Parameterized Macromodeling Strategy with Uniform Stability Test", IEEE Trans. on Advanced Packaging, vol. 32, no. 1, pp. 205-215, February 2009.

[4] F. Ferranti, L. Knockaert, T. Dhaene, "Passivity-preserving Interpolationbased Parameterized Macromodeling of Scattered S-data", Microw. and Wireless Comp. Lett., vol. 20, no. 3, pp. 133-135, March 2010.

[5] S. Lefteriu and H. Mohring, "Generating Parametric Models from Tabulated Data", IEEE Design Autom. Conf., pp. 679-682, June 2010.

[6] P. Triverio, M. S. Nakhla, S. Grivet-Talocia, "Passive Parametric Macromodeling from Sampled Frequency Data", IEEE Workshop Signal Prop. on Interconnects, pp.1-4, May 2010.

[7] J. P. Berrut and L. N. Trefethen, "Barycentric Lagrange Interpolation", SIAM Review, vol. 46, no. 3, pp. 501-517, pp. 501-517, September 2004.

[8] B. Gustavsen and A. Semlyen, "Rational approximation of frequency domain responses by vector fitting", IEEE Trans. on Power Delivery, vol. 14, no. 3, pp. 1052-1060, July 1999.

[9] C. Saunders, J. Hu, C. E. Christoffersen, M. B. Steer, "Inverse Singular Value Method for Enforcing Passivity in Reduced-Order Models of Distributed Structures for Transient and Steady-State Simulation", IEEE Trans. on Microw. Theory Tech., vol. 59, no. 4, pp. 837-847, April 2011.

[10] F. Ferranti, L. Knockaert, T. Dhaene, G. Antonini, "Passivity-Preserving Parametric Macromodeling for Highly Dynamic Tabulated Data Based on Lure Equations", IEEE Trans. Microw. Theory Tech., vol. 58, no. 12, pp. 3688-396, December 2010. 